\title{
Frequency of Discordance between Facet Joint Activity on Technetium Tc99m Methylene Diphosphonate SPECT/CT and Selection for Percutaneous Treatment at a Large Multispecialty Institution
}

\author{
V.T. Lehman, R.C. Murphy, T.J. Kaufmann, F.E. Diehn, N.S. Murthy, J.T. Wald, K.R. Thielen, K.K. Amrami, J.M. Morris, and T.P. Maus
}

\begin{abstract}
BACKGROUND AND PURPOSE: The clinical impact of facet joint bone scan activity is not fully understood. The hypothesis of this study is that facet joints targeted for percutaneous treatment in clinical practice differ from those with reported activity on technetium Tc99m methylene diphosphonate SPECT/CT.
\end{abstract}

\begin{abstract}
MATERIALS AND METHODS: All patients with a technetium Tc99m methylene diphosphonate SPECT/CT scan of the lumbar or cervical spine who underwent subsequent percutaneous facet joint steroid injection or comparative medial branch blocks at our institution between January 1, 2008, and February 19, 2013, were identified. Facet joints with increased activity were compared with those treated. A chart review characterized the clinical reasons for treatment discrepancies.
\end{abstract}

RESULTS: Of 74 patients meeting inclusion criteria, 52 (70\%) had discrepant imaging findings and treatment selection of at least 1 facet joint, whereas 34 patients (46\%) had a side (right vs left) discrepancy. Only 92 (70\%) of 132 facet joints with increased activity were treated, whereas 103 (53\%) of 195 of treated facet joints did not have increased activity. The most commonly documented clinical rationale for discrepancy was facet joint activity that was not thought to correlate with clinical findings, cited in 18 (35\%) of 52 patients.

CONCLUSIONS: Facet joints undergoing targeted percutaneous treatment were frequently discordant with those demonstrating increased technetium Tc99m methylene diphosphonate activity identified by SPECT/CT at our institution, in many cases because the active facet joint(s) did not correlate with clinical findings. Further prospective double-blinded investigations of the clinical significance of facet joint activity by use of technetium Tc99m methylene diphosphonate SPECT/CT and comparative medial branch blocks are needed.

ABBREVIATIONS: ${ }^{99 \mathrm{~m}} \mathrm{Tc}$ MDP = technetium Tc99m methylene diphosphonate; $\mathrm{RF}=$ radiofrequency

C linical examination and anatomic imaging do not reliably identify specific painful facet joints. ${ }^{1-3}$ Prior studies suggest that technetium Tc99m methylene diphosphonate ( ${ }^{99 m}$ Tc MDP) bone scan activity can predict if a facet joint is painful and if there will be a positive response to percutaneous intervention targeted specifically to active facet joints. ${ }^{4-6}$ These prior studies used strict treatment of every facet joint with increased radiotracer activity on bone scan, ${ }^{4-6}$ concluding that ${ }^{99 \mathrm{~m}}$ Tc MDP SPECT can decrease the number of treated facet joints. ${ }^{4}$ However, those results can only be applicable if this is used in clinical practice and is feasible. Moreover, these studies did not incorporate CT scan for facet joint localization, use comparative medial branch blocks for diagnosis, include clearly blinded patients and proceduralists, or ex-

Received May 14, 2013; accepted after revision July 8.

From the Department of Radiology, Mayo Clinic College of Graduate Medical Education, Rochester, Minnesota.

Please address correspondence to: Vance T. Lehman, Department of Radiology, 200 First St SW, Rochester, MN 55905; e-mail: lehman.vance@mayo.edu

http://dx.doi.org/10.3174/ajnr.A3731 amine the impact of ${ }^{99 \mathrm{~m}} \mathrm{Tc}$ MDP bone scan results in actual clinical practice. Such shortcomings limit the conclusions of these prior reports and indicate the need for further examination of the clinical usefulness of ${ }^{99 \mathrm{~m}} \mathrm{Tc}$ MDP facet joint activity. In addition, evaluation of the impact of imaging in actual practice is important because the efficacy demonstrated in clinical studies often does not translate into true clinical effectiveness when the ideal rigorous methods of the efficacy study are no longer applied. ${ }^{7}$ That is, the effect of real-life variables such as clinical findings suggesting facet joint pain on a specific side or level and the variability of physician experience and philosophy should be evaluated to confirm or refute the impact of controlled studies on actual clinical practice and to identify areas in need of future investigation.

In our anecdotal experience, the specific facet joints referred for percutaneous facet joint intervention are sometimes widely discordant from those with reported activity on ${ }^{99 \mathrm{~m}} \mathrm{Tc}$ MDP SPECT/CT. Specifically, we have seen some patients with suspected facetogenic pain where the ${ }^{99 \mathrm{~m}} \mathrm{Tc}$ MDP SPECT/CT scan does not seem to demonstrate concordant facet joint activity in 
the location or even on the side of pain. We have also observed that many facet joints with bone scan activity do not seem to correlate with a clinical facet joint pain syndrome. However, the concordance of facet joint bone scan activity and targeted facet joint treatment in actual clinical practice is not well described. Furthermore, the clinical rationale for imaging-procedural discrepancy is not known.

The hypothesis of this study is that facet joints that are targeted for percutaneous treatment in clinical practice differ from those with reported activity on ${ }^{99 m}$ Tc MDP SPECT/CT scans.

\section{MATERIALS AND METHODS}

\section{Patients}

Approval for this Health Information Portability and Accountability compliant study was obtained from our institutional review board.

A chart review was performed of all patients who underwent both ${ }^{99 \mathrm{~m}} \mathrm{Tc}$ MDP SPECT/CT imaging of the cervical or lumbar spine and percutaneous facet joint intervention (facet joint injection or medial branch block) between January 1, 2008, and February 19, 2013, at our institution.

Exclusion criteria were as follows: 1) cases in which the interval between the ${ }^{99 \mathrm{~m}} \mathrm{Tc}$ MDP SPECT/CT examination and facet joint injection exceeded 100 days, and 2) cases in which facet joints with increased ${ }^{99 \mathrm{~m}}$ Tc MDP activity were not specifically named by level and side in the radiology report (eg, a statement such as "scattered facet joint activity" or "lower lumbar facet joint activity").

\section{${ }^{99 m}$ Tc MDP SPECT/CT Examinations}

Patients underwent SPECT/CT of the cervical or lumbar spine 3-4 hours after injection with $20 \mathrm{mCi}( \pm 10 \%){ }^{99 \mathrm{~m}} \mathrm{Tc} \mathrm{MDP}$. Examinations were performed on a Precedence 6-section or a 16section scanner (Skylight SPECT system and Brilliance CT scanner; Philips Healthcare, Best, the Netherlands). SPECT parameters were as follows: $128 \times 128$ word mode matrix, 128 views at 20 seconds per view, 1.46 zoom factor, step-and-shoot angular step of $3^{\circ}$, body contouring, and low-energy all-purpose collimator. CT parameters were as follows: $120 \mathrm{kVp}, 60 \mathrm{mAs}$ per section, 3-mm section thickness, and 3-mm increment. CT studies were acquired with helical scans with a pitch of 0.788 (6-section) or 0.813 (16-section) and a collimator thickness of $3 \mathrm{~mm}$ (6-section) and $1.5 \mathrm{~mm}$ (16-section).

\section{Chart Review of SPECT/CT Reports and Treated Facet Joints}

All relevant clinical notes between the time of ${ }^{99 \mathrm{~m}} \mathrm{Tc}$ MDP SPECT/CT examinations and facet joint interventions, the ${ }^{99 \mathrm{~m}} \mathrm{Tc}$ MDP SPECT/CT reports, the ${ }^{99 \mathrm{~m}}$ Tc MDP SPECT/CT images, the facet joint intervention procedure reports, and the procedural fluoroscopy images were reviewed in detail by a board-certified radiologist (V.T.L.) for side and level confirmation. All images were viewed on an Advantage workstation (GE Healthcare, Milwaukee, Wisconsin). Because the aim of our study was to characterize selection of treated facet joints in actual clinical practice rather than patient outcome, we did not perform de novo characterization of facet joint activity and success of facet joint injection (intra-articular vs periarticular). The specialty of the refer- ring clinical provider was recorded. Cases in which the referring provider differed from the provider who ordered the ${ }^{99 \mathrm{~m}} \mathrm{Tc}$ MDP SPECT/CT were noted; in these cases, the clinical notes of the referring provider were examined.

In the cervical spine, the bilateral C1-2 articulations through the C7-T1 facet joints were assessed (totaling 14 cervical facet joints for each patient). In the lumbar spine, the bilateral T12-L1 through the L5-S1 facet joints were assessed (totaling 12 facet joints for each patient). Because the number of lumbar vertebral bodies is variable, the lowest presacral lumbar-type vertebral body was designated L5 for the purposes of this analysis.

The facet joints targeted for percutaneous intervention, method of intervention (steroid injection or medial branch block), and type of image guidance were recorded. Although medial branch blocks are diagnostic procedures usually performed in 2 sessions to determine the appropriateness of subsequent radiofrequency $(\mathrm{RF})$ ablation at our institution, the facet joints targeted with the first medial branch block were used for this portion of the analysis for consistency.

\section{Patient Referral Practice Model}

Our institution is a large, multispecialty, tertiary referral center and includes a dedicated spine center with a spine clinic and a spine interventional practice. The spine interventional practice is staffed by fellowship-trained musculoskeletal radiologists, neuroradiologists, physical medicine and rehabilitation physicians, and anesthesiologists with subspecialty training in pain management. Specific facet joints targeted by the interventional practice are generally requested by referring clinicians. Occasionally, the proceduralist will choose different facet joints to target after conferring with the referring clinician. Referrals commonly come from pain medicine, though a wide variety of clinicians such as neurologists or primary care physicians also provide referrals. Facet joint interventions may be either steroid injections or RF ablation after dual comparative medial branch blocks, though facet joint injections are used more frequently at our institution. No specified criteria exist for selection of medial branch blocks with RF ablation; these are chosen based on clinical factors such as the preference of the specific referring provider and the expertise of the requested proceduralist. The specialty of the proceduralist for each intervention was recorded.

\section{Data Analysis}

A grouped data analysis was performed for all included patients. Subanalyses for the lumbar spine and cervical spine were also performed. The presence or absence of reported increased activity within each facet joint was compared with the presence or absence of targeted treatment of that facet joint. Facet joints were placed into 3 categories: 1 ) facet joints with reported ${ }^{99 \mathrm{~m}} \mathrm{Tc}$ MPD activity and that were also treated (concordant), 2) facet joints with reported ${ }^{99 \mathrm{~m}} \mathrm{Tc}$ MDP activity and that were not targeted for treatment (discordant), and 3) treated facet joints that were not reported as having ${ }^{99 \mathrm{~m}} \mathrm{Tc}$ MDP activity (discordant). Patients were also categorized as either having 1) concordance of activity and injection for every facet joint or 2) at least 1 discordant facet joint. Facet joints without increased radiotracer activity that were not treated were also considered concordant, but further 


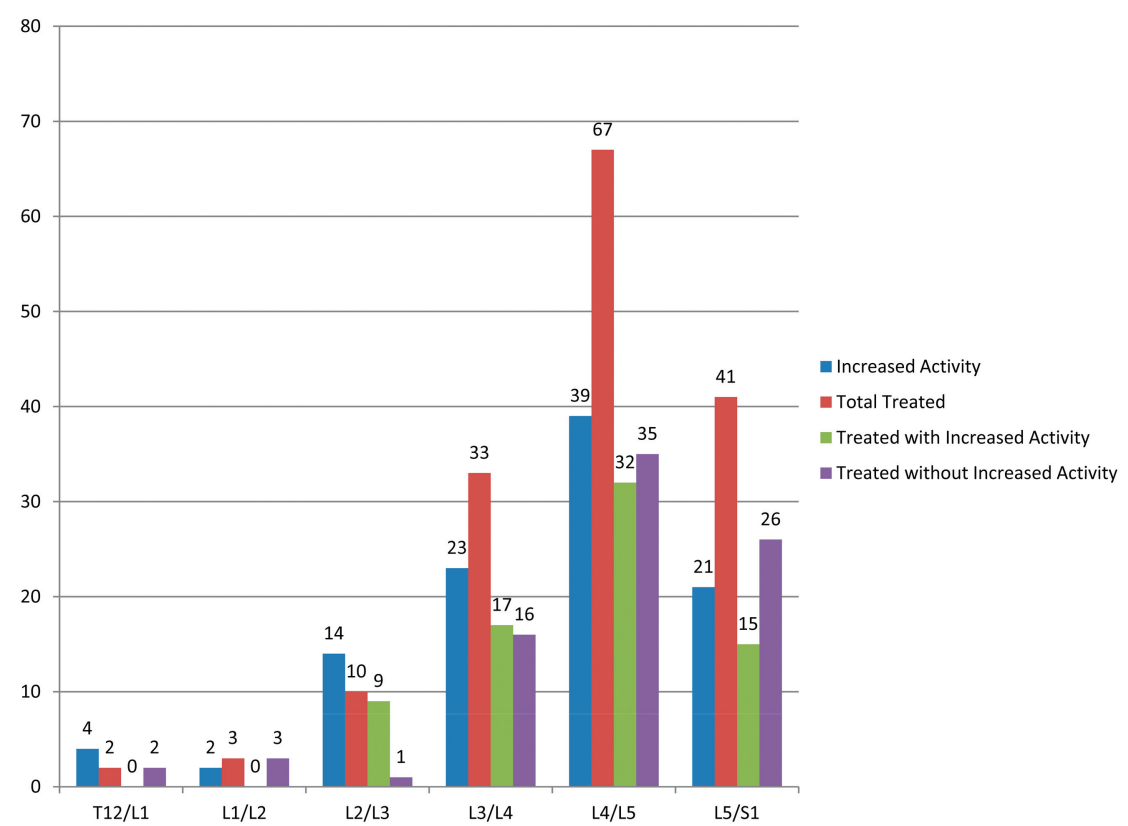

FIG 1. Number of lumbar facet joints per level with reported increased activity, total treated at that level with breakdown of those treated with and without activity. The left and right facet joints are grouped at each level.

detailed analysis of this category was not performed because a large number of facet joints that would not have been considered for treatment with or without imaging likely fall within this group.

The degree of left-right concordance of facet joint interventions and activity was recorded. Specifically, it was determined for each patient if 1 ) there was at least 1 injection on a side without a single facet joint with increased activity and/or, 2) there was a side with at least 1 facet joint with increased activity but without a single injection.

\section{Categorization of Rationale for ${ }^{99 m}$ TC MDP SPECT/CT-Procedural Discrepancy}

In cases with requests for facet joint intervention that were discordant with the ${ }^{99 \mathrm{~m}} \mathrm{Tc}$ SPECT/CT results in at least 1 facet joint, the clinical rationales for the discrepancies were categorized as follows: 1) facet joints with activity that did not correlate with clinical examination, 2) consideration of positive or negative response of some facet joints to prior intervention, 3) de-emphasis of facet joints with relatively low reported activity, 4) too many facet joints with activity to include each one for treatment, 5) targeted facet joints selected based on anatomic features of facet joint degenerative change or information obtained from other imaging test such as MR imaging, and 6) no documented clinical reasoning identified. Cases with multiple cited categories were grouped into the single predominant category cited in the medical record.

\section{RESULTS}

\section{Patients, Referring Clinicians, and ${ }^{99 m}$ Tc MDP SPECT/CT Examinations}

A total of 78 patients with a ${ }^{99 \mathrm{~m}} \mathrm{Tc}$ MDP SPECT/CT and subsequent facet joint injection were identified. Overall, 4 were excluded for the following reasons: nonspecific reporting of facet joint activity in the radiology report $(n=3)$, and $>100$-day inter- val between imaging and intervention $(n=1)$. Therefore, 74 patients were included in this study, consisting of 43 women and 31 men with a mean age of 59 years (age range, 28-93 years). No included patient had a ${ }^{99 \mathrm{~m}} \mathrm{Tc}$ MDP SPECT/CT and intervention of both the lumbar and cervical spine. Fifty-five patients (74\%) underwent ${ }^{99 \mathrm{~m}} \mathrm{Tc}$ MDP SPECT/CT and injection of the lumbar spine; 19 patients (26\%), of the cervical spine. A total of 926 facet joints were assessed in this study: 660 in the lumbar spine and 266 in the cervical spine. The mean time between the ${ }^{99 \mathrm{~m}} \mathrm{Tc}$ MDP SPECT/CT and the percutaneous intervention was 15.6 days (range, 0-99 days) overall, 13.4 days (range, 0-99 days) for the lumbar spine, and 22.1 days (range, 0-65 days) for the cervical spine.

The clinicians who ordered the ${ }^{99 \mathrm{~m}} \mathrm{Tc}$ MDP SPECT/CT by specialty were physical medicine and rehabilitation $(n=48$; $65 \%)$, pain medicine $(n=10 ; 14 \%)$, neurology $(n=7 ; 9 \%)$, orthopedics $(n=4 ; 5 \%)$, primary care $(n=3$; $4 \%)$, and rheumatology $(n=2 ; 3 \%)$. Pain medicine includes physicians with either a physical medicine and rehabilitation or anesthesiology background. All $74{ }^{99 \mathrm{~m}} \mathrm{Tc}$ MDP SPECT/CT examinations (100\%) were originally performed with an indication to identify a pain generator rather than for a different category of diagnosis, such as metastatic disease or infection evaluation. Specifically, of the 74 examinations performed, 43 (58\%) had an indication for facet joint evaluation, $23(31 \%)$ were ordered to identify a pain generator with a general indication of "low back pain," $6(8 \%)$ were ordered to identify a pain generator with a general indication of "neck pain," 1 (1\%) was ordered to evaluate for possible fracture, and 1 ( $1 \%$ ) was ordered to evaluate for a possible pars interarticularis defect.

Of 74 facet joint treatment referrals, 64 (86\%) were requested directly from the physician who officially requested the ${ }^{99 \mathrm{~m}} \mathrm{Tc}$ MDP SPECT/CT. Of the remaining 10 (14\%) patients, facet joint injections were requested via an intermediary pain medicine specialist for 6 patients and an intermediary physical medicine and rehabilitation specialist in 4 patients.

\section{Facet Joint Activity and Percutaneous Treatment}

Overall, 132 facet joints (14\%) had increased activity, 103 (16\%) of 660 in the lumbar spine and $29(11 \%)$ of 266 in the cervical spine. A total of 195 facet joints (21\%) underwent percutaneous treatment under fluoroscopy: 156 (23\%) in the lumbar spine and 39 (15\%) in the cervical spine. The most common lumbar and cervical levels with both facet joint activity $(n=39)$ and intervention were L4-5 $(n=67)$ and C3-4 $(n=11)$, respectively. Overall, 67 (91\%) of 74 patients underwent steroid injection: 49 (89\%) in the lumbar spine and 18 $(95 \%)$ in the cervical spine. Seven patients (9\%) underwent medial branch block: $6(11 \%)$ in the lumbar spine and $1(5 \%)$ in the cervical spine. The rate of reported concordant/discordant facet joint activity and treatment at each level is depicted in Figs 1 and 2. 
On a per-patient basis, 52 patients $(70 \%)$ had at least 1 discrepancy between reported facet joint activity and treatment, whereas $22(30 \%)$ had no discrepancy, including 14 with lumbar spine intervention and 8 with cervical spine intervention. The rates of discrepancy per patient according to specialty of provider who directly ordered the facet joint injection were as follows: 1 ) physical medicine and rehabilitation (34 [69\%] of 49), 2) pain medicine (10 [66\%] of 16), 3) neurology (3 [100\%] of 3), 4) adult primary care $(2[100 \%]$ of 2$), 5)$ orthopedic surgery $(2[66 \%]$ of 3 ), and 6) rheumatology (1 [100\%] of 1).

On a per-facet basis, 92 (47\%) of 195 treated facet joints had increased activity on the ${ }^{99 \mathrm{~m}}$ Tc MDP SPECT/CT, whereas 103 targeted fact joints (53\%) did not have increased activity. Therefore, of the 132 facet joints with increased activity, 92 (70\%) were treated and $40(30 \%)$ were not. The breakdown of the rate of facet joint intervention by activity and anatomic region is listed in Table 1.

Overall, 34 (46\%) of 74 patients had a right-left side discrepancy. Specifically, 11(15\%) of 74 had activity on 1 side that was not treated, and 24 (32\%) of 74 patients had treatment to at least 1 side that did not have increased activity. One patient was included in both of these groups. Clinical examples of patients with discordant treatment are depicted in Figs 3 and 4.

\section{Clinical Reasons for ${ }^{99 m}$ TC MDP SPECT/CT and Procedure Discordance}

A total of 31 (60\%) of 52 patients with a discrepancy had a documented clinical reason, whereas 21 (40\%) did not. The most com-

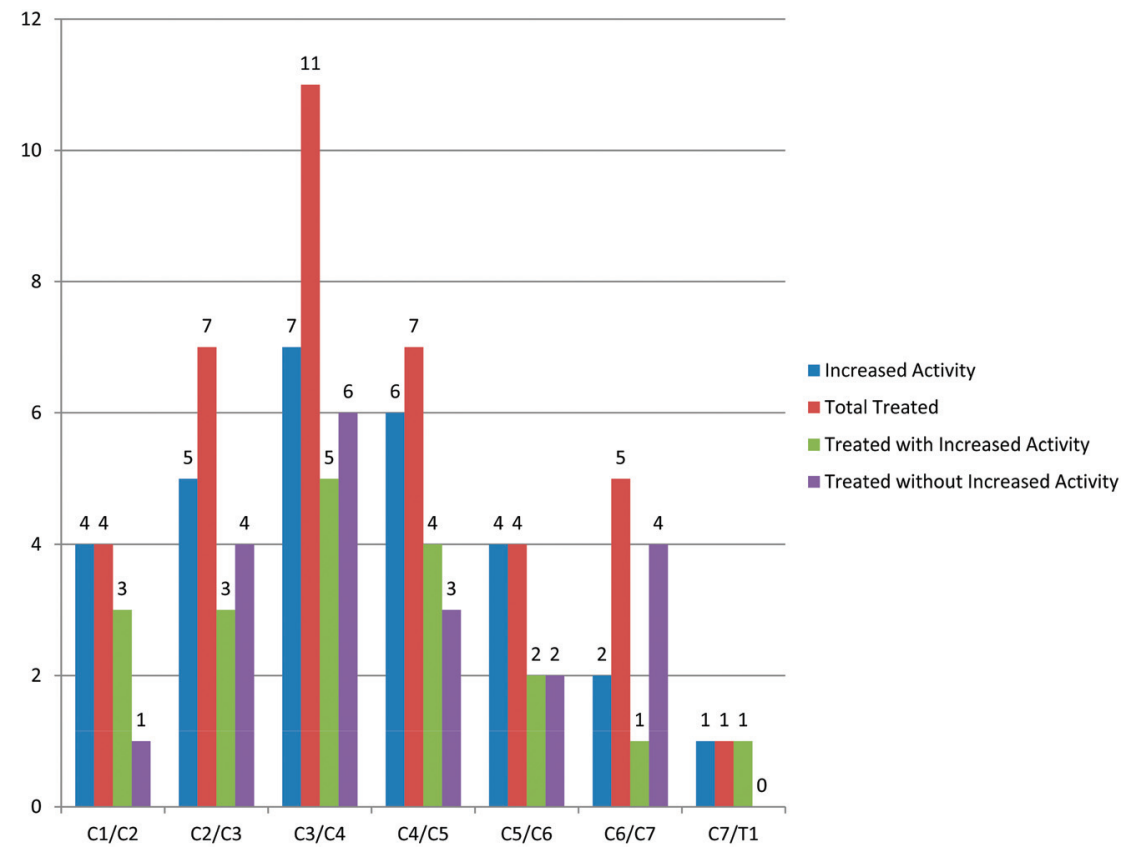

FIG 2. Number of cervical facet joints per level with reported increased activity, total treated at that level with breakdown of those treated with and without activity. The left and right facet joints are grouped at each level. mon cited reason was that the ${ }^{99 \mathrm{~m}} \mathrm{Tc}$ MDP activity did not correspond with the clinical findings, documented in 18 (35\%) of 52 patients. The reasons for discordance are detailed in Table 2.

\section{DISCUSSION}

The results of our study are that in the clinical practice at a large multispecialty institution, targeted percutaneous facet joint treatments were discrepant from ${ }^{99 \mathrm{~m}} \mathrm{Tc}$ MDP SPECT/CT activity in most patients, many facet joints with increased activity were not targeted, many targeted facet joints did not demonstrate increased activity, and facet joint activity did not always correlate with clinically suspected painful facet joints or even the side of suspected painful facet arthropathy. These findings have implications for patient treatment and the need for further evaluation of the significance, accuracy, and predictive ability of ${ }^{99 \mathrm{~m}} \mathrm{Tc}$ MDP SPECT/CT findings in evaluation of facet joint pain.

Prior reports have found that strictly targeting facet joints with increased ${ }^{99 \mathrm{~m}} \mathrm{Tc}$ MDP activity is predictive of a positive response to percutaneous treatment ${ }^{4-6}$ and that use of bone scans can decrease the number of treated facet joints. ${ }^{4}$ However, in the current retrospective review of our institution, this association did not translate into actual clinical practice for patients in whom ${ }^{99 \mathrm{~m}} \mathrm{Tc}$ MDP SPECT/CT examinations were ordered specifically to identify pain generators, mostly by pain physicians. The most commonly found documented reason was lack of correlation of facet joints with increased activity and clinical findings. Therefore, it is possible that the requesting providers were unable to reconcile these apparent discrepancies and, when discordant, instead requested treatment of clinically implicated facet joints. For clinicians to reconcile widely discrepant imaging and clinical indicators, strong evidence guiding the usefulness, accuracy, and impact on patient outcomes is desirable. However, the lack of prior studies that fully meet current practice standards in conjunction with the results of our current study suggests that the significance of facet joint activity with ${ }^{99 \mathrm{~m}} \mathrm{Tc}$ is not yet fully understood.

Prior studies were performed with ${ }^{99 \mathrm{~m}}$ Tc SPECT only, ${ }^{4-6}$ rather than ${ }^{99 \mathrm{~m}} \mathrm{Tc}$ SPECT/CT for anatomic co-localization to definitively confirm the correct level of activity. Anatomic co-localization is important because facet joints are anatomically juxtaposed, the number of vertebral bodies is variable, and transitional lumbosacral vertebral bodies are present in $4 \%-30 \%$ of patients. ${ }^{8}$ Previously, criteria for active facet joints were either not

Table 1: Facet joint ${ }^{99 \mathrm{~m}} \mathrm{Tc}$ MDP activity, percutaneous treatment, and anatomic region

\begin{tabular}{|c|c|c|c|c|c|c|}
\hline & \multicolumn{3}{|c|}{ Facet Joints Treated Percutaneously } & \multicolumn{3}{|c|}{ Facet Joints Not Treated Percutaneously } \\
\hline & Lumbar $(n=156)$ & Cervical $(n=39)$ & Overall $(n=195)$ & Lumbar $(n=504)$ & Cervical $(n=227)$ & Overall $(n=731)$ \\
\hline Activity present (\%) & $73(47)$ & $19(49)$ & $92(47)$ & $30(6)$ & $10(4)$ & $40(5)$ \\
\hline Activity absent (\%) & $83(53)$ & $20(51)$ & $103(53)$ & 474 (94) & $217(96)$ & $691(95)$ \\
\hline
\end{tabular}



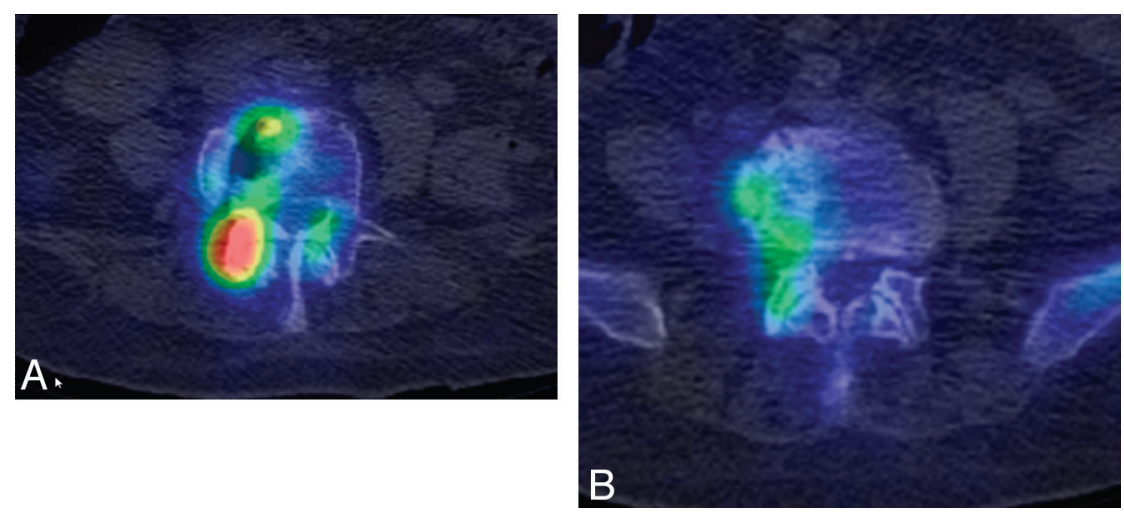

FIG 3. Left-right discordance with treatment of side with no reported activity in addition to a side with activity in a 90 -year-old woman with bilateral low back pain. ${ }^{99 \mathrm{~m}} \mathrm{TC}$ MDP SPECT/CT demonstrates activity at the right, but not left, L3/L4 facet joint $(A)$, and no reported increased activity at the bilateral L4/L5 facet joints $(B)$ or other lumbar facet joints. Because of severe bilateral low back pain attributed to facet arthropathy, she underwent bilateral L3/L4 and L4/L5 facet joint injections.
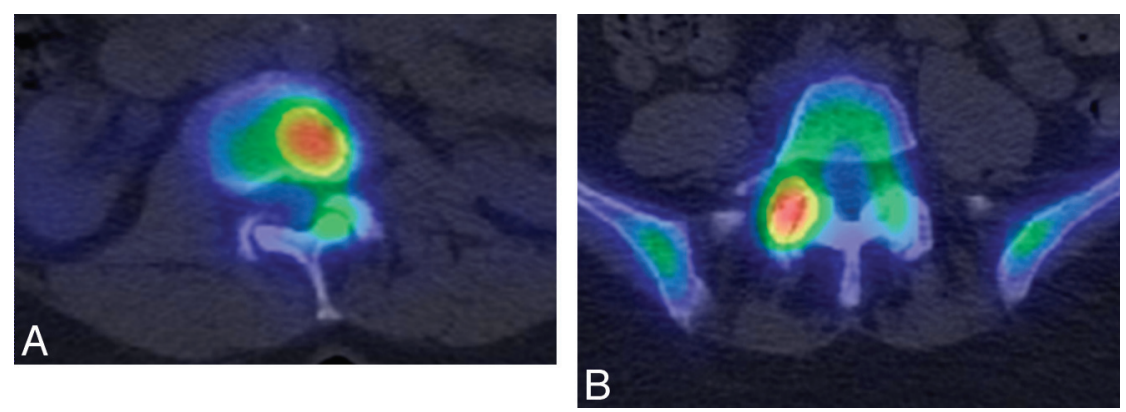

FIG 4. Left-right discordance with absent treatment on a side with activity as well as level discordance in a 60-year-old woman with back pain and clinical findings supporting a left upper facet joint pain generator. Mildly increased activity in the left L1-L2 facet joint $(A)$ and marked increased activity in the right $\mathrm{L} 4-\mathrm{L} 5$ facet joint $(B)$. Only the left L1-L2 facet joint underwent facet joint steroid injection.

Table 2: Reasons for discrepancy between facet joint activity and treatment

\begin{tabular}{lccc}
\hline \multirow{2}{*}{\multicolumn{1}{c}{ Reason }} & \multicolumn{3}{c}{ Patient Group } \\
\cline { 2 - 4 } & $\begin{array}{c}\text { Lumbar } \\
(\boldsymbol{n}=\mathbf{4 1})\end{array}$ & $\begin{array}{c}\text { Cervical } \\
(\boldsymbol{n}=\mathbf{1 1})\end{array}$ & $\begin{array}{c}\text { Overall } \\
(\boldsymbol{n}=\mathbf{5 2})\end{array}$ \\
\hline Not documented (\%) & $18(44)$ & $3(27)$ & $21(40)$ \\
Active facet joint not concordant with clinical findings (\%) & $13(32)$ & $5(45)$ & $18(35)$ \\
Consideration of response (or lack of) to prior targeted & $6(15)$ & $3(27)$ & $9(17)$ \\
treatment (\%) & & & \\
Relatively low reported activity (\%) & $2(5)$ & $0(0)$ & $2(4)$ \\
Too many active facet joints to inject (\%) & $0(0)$ & $0(0)$ & $0(0)$ \\
Information from other imaging (\%) & $2(5)$ & $0(0)$ & $2(4)$ \\
\hline
\end{tabular}

clearly defined ${ }^{5-6}$ or were arbitrarily designated as those greater than the adjacent vertebral body, ${ }^{4}$ which could be problematic because vertebral body activity at the levels of degenerative spinal disease is highly variable in our experience. In addition, prior studies have included patients and proceduralists who were not clearly blinded, ${ }^{4-6}$ had widely overlapping statistical error measurements between treatment and control groups, ${ }^{4}$ and/or used facet joint injections/single medial branch blocks. ${ }^{4-6}$ A prior case series analysis describes a positive response to treatment of facet joints selected with ${ }^{99 \mathrm{~m}} \mathrm{Tc}$ SPECT/CT where the patients and proceduralists were not clearly blinded, there was no control group, and comparative medial branch blocks were not used. ${ }^{9}$ Although facet joint injections remain a treatment option in routine clinical practice, these have a $32 \%$ placebo rate. ${ }^{10}$ Comparative medial branch blocks are now considered the reference standard for diagnosis of painful facet joints and should be used for rigorous prospective study that will be fully accepted by the pain medicine community. ${ }^{11}$

There are many possible explanations for the results of our study, though these are speculative given the retrospective study design. ${ }^{99 \mathrm{~m}} \mathrm{Tc}$ MDP facet joint activity may not be necessary for or provide sufficient evidence of a painful facet joint. That is, facet joint activity is dependent on vascularity and chemisorption into bone mineral, particularly during osteoneogenesis, ${ }^{12}$ and therefore has multiple potential causes such as active growth of an osteophyte $^{13}$ or inflammation. ${ }^{4,14}$ These may not be equally associated with pain. In addition, the pathomechanism of facet joint pain is not fully understood and is likely multifactorial. ${ }^{15}$ It is possible that bone scan activity is present with some, but not all, causes or predisposing factors of facet joint pain. Clinicians may have also, knowingly or unknowingly, considered the reported prevalence of facet joint pain at specific spinal levels in decision making, which would be consistent with the finding that many facet joints without activity that were treated were at the L4-5 level. ${ }^{16}$ Because clinical localization of facet joint pain is challenging, it is possible that some cases had false assignment of discordant facet joint activity and clinical findings. However, the cases of complete side discrepancy indicate that false clinical localization is unlikely in all discordant cases; note that a prior study found no instances of contralateral facet joint pain referral. ${ }^{17}$ Finally, it is possible that patients who underwent ${ }^{99 \mathrm{~m}} \mathrm{Tc}$ MDP SPECT/CT were a subgroup with confusing or challenging clinical findings and were not fully representative of all patients with facetogenic back pain.

Because our institution did not collect outcome data in a consistent standardized format for all patients during the period of this retrospective study, lack of outcome assessment was a limitation. However, outcome data derived from patients treated primarily with steroid injections would have had limited usefulness because reliable determination of facet joint pain depends on consistent use of diagnostic comparative medial branch blocks. ${ }^{11}$ Strict use of comparative medial branch blocks would likely require a prospective study design, ideally randomized and double blinded. Even with this limitation, the results of this study do enhance clinical equipoise for such a future 
investigation because the effectiveness of 99mTc MDP SPECT/CT for prescription of treatment of specific facet joints in actual clinical practice was not high.

Our study had several other limitations. Because the patients were treated at a single institution, it is uncertain how generalizable these results were, and corroboration of results from other institutions would strengthen the findings. The retrospective study design precluded standardized patient recruitment, clinical assessment of all patients, consistent auditing of clinical decision making, and assessment of the efficacy of facet joint treatments. The included patients were assessed by a variety of clinicians who may have had different varying approaches to the use of bone scan activity in clinical decision making. The ${ }^{99 \mathrm{~m}} \mathrm{Tc}$ MDP examinations were interpreted by a variety of nuclear medicine physicians who might have had varied practice patterns and varied thresholds for reporting or intervening on imaging findings. There is no commonly used clinical grading scale of facet joint activity, and our study dichotomized activity on the basis of only the radiology reports, reflecting actual clinical practice. A small number of facet joint injections were ordered by physicians who did not originally order or reference the bone scan.

In addition, future research could evaluate the significance of degree of facet joint activity and could evaluate if other clinical or radiographic findings are useful when used in conjunction with ${ }^{99 \mathrm{~m}} \mathrm{Tc}$ MDP SPECT/CT results. It would also be useful to establish the prevalence of facet joint activity in patients without axial low back pain to assess specificity.

\section{CONCLUSIONS}

Facet joints undergoing targeted percutaneous treatment were frequently discordant with those demonstrating increased ${ }^{99 \mathrm{~m}} \mathrm{Tc}$ MDP activity identified by SPECT/CT at our institution, often because the active facet joints did not seem to correlate with clinical findings. Further prospective, double-blinded investigation of the clinical significance of facet joint activity by use of ${ }^{99 \mathrm{~m}} \mathrm{Tc}$ MDP SPECT/CT and comparative medial branch blocks is needed.

Disclosures: Kent Thielen—UNRELATED: Royalties: Nevro.* Timothy Maus—OTHER RELATIONSHIPS: Board of Directors, International Spine Intervention Society. *Money paid to institution

\section{REFERENCES}

1. Bogduk N. Degenerative joint disease of the spine. Radiol Clin North Am 2012;50:613-28
2. Cohen SP, Raja SN. Pathogenesis, diagnosis, and treatment of lumbar zygapophysial (facet) joint pain. Anesthesiology 2007;106: 591-614

3. Siegenthalar A, Eichenberger U, Schmidlin K, et al. What does local tenderness say about the origin of pain? An investigation of cervical zygapophysial joint pain. Anesth Analg 2010;110:923-27

4. Pneumaticos SP, Chatziioannou SN, Hipp JA, et al. Low back pain: prediction of short-term outcome of facet joint injection with bone scintigraphy. Radiology 2006;238:693-98

5. Dolan AL, Ryan PJ, Arden NK, et al. The value of SPECT scans in identifying back pain likely to benefit from facet joint injection. $\mathrm{BrJ}$ Rheumatol 1996;35:1269-73

6. Koh WU, Kim SH, Hwang BY, et al. Value of bone scintigraphy and single photon emission tomography (SPECT) in lumbar facet disease and prediction of short-term outcome of ultrasound guided medial branch block with bone SPECT. Korean J Pain 2011; 24:81-86

7. Sunshine JH, Applegate KE. Technology assessment for radiologists. Radiology 2004;230:309-14

8. Konin GP, Walz DM. Lumbosacral transitional vertebrae: classification, imaging findings, and clinical relevance. AJNR Am J Neuroradiol 2010;31:1778-86

9. McDonald M, Cooper R, Wang MY. Use of computed tomographysingle-photon emission computed tomography fusion for diagnosing painful facet arthropathy. Technical note. Neurosurg Focus 2007;22:E2

10. Schwarzer AC, Wang S, BogdukN, et al. Prevalence and clinical features of lumbar zygapophyseal joint pain: a study in an Australian population with chronic low back pain. Ann Rheum Dis 1995; 54:100-06

11. Bogduk N, ed. Practice Guidelines for Spinal Diagnostic and Treatment Procedures. 2nd ed. San Francisco: International Spine Intervention Society; 2004, pp. 559-63

12. Fogelman I. Skeletal uptake of disophosphonate: a review. Eur J Nucl Med 1980;5:473-76

13. Christensen SB. Localization of bone-seeking agents in developing experimentally induced osteoarthritis in the knee joint of the rabbit. Scand J Rheumatol 1983;12:343-49

14. Cavanaugh JM, Ozaktay C, Yamashita T, et al. Mechanisms of low back pain: a neurophysiologic and neuroanatomic study. Clin Orthop Relat Res 1997;335:166-80

15. Bogduk N. Clinical and Radiological Anatomy of the Lumbar Spine, 5th ed. St. Louis: Elsevier; 2012:183-86

16. Eubanks JD, Lee MJ, Cassinelli E, et al. Prevalence of lumbar facet arthrosis and its relationship to age, sex, and race: an anatomic study of cadaveric specimens. Spine 2007;32:2058-62

17. McCall IW, Park WM, O'Brien JP. Induced pain referral from posterior lumbar elements in normal subjects. Spine 1979;4: 441-46 